\title{
The ratio of small to large separations of stellar p-modes
}

\author{
I. W. Roxburgh ${ }^{1,2}$ \\ 1 Astronomy Unit, Queen Mary, University of London, Mile End Road, London E1 4NS, UK \\ e-mail: i.w.roxburgh@qmul.ac.uk \\ 2 LESIA, Observatoire de Paris, Place Jules Janssen, 92195 Meudon, France
}

Received 6 September 2004 / Accepted 23 December 2004

\begin{abstract}
In previous papers we showed that the ratios of the small to large separations of acoustic oscillations in solar-like stars are approximately independent of the structure of the outer layers, and are therefore diagnostics of the interior structure alone. Here we present similar results for a range stellar models of mass $0.8,1.0,1.2,1.5,2,10 M_{\odot}$, all of which show this same property.
\end{abstract}

Key words. stars: oscillations - stars: interiors

\section{Introduction}

In previous papers (Roxburgh 2004; Roxburgh \& Vorontsov 2003) we demonstrated that the ratios of the small to large separations of acoustic oscillations in solar-like stars are approximately independent of the structure of the outer layers, and are therefore diagnostics of the interior structure alone. This was done empirically by showing that these ratios remained unaltered when the structure of the outer layers of a solar model was changed, and theoretically by demonstrating that these ratios are approximately given by the internal phase shifts of the acoustic oscillations.

The examples given in previous work were only for modified solar models; we here present similar results for models of main sequence stars with masses ranging from $0.8 M_{\odot}$ to $10 M_{\odot}$. In the first experiment we replace the outer $10 \%$ of the radius of the stellar model by a uniform polytropic structure, and in the second experiment simply chop off the outer layers at radii of $85 \%, 90 \%$ and $95 \%$ of the stellar radius. For all models the ratios of small to large separations are approximately independent of the structure of the outer layers.

The large separations $\Delta_{0}, \Delta_{1}$, small separations of even parity $d_{02}, d_{13}$, and their ratios $r_{02}, r_{13}$, are defined as

$$
\begin{aligned}
& \Delta_{0}(n)=v_{n, 0}-v_{n-1,0} \quad \Delta_{1}(n)=v_{n, 1}-v_{n-1,1} \\
& d_{02}(n)=\left(v_{n, 0}-v_{n-1,2}\right) \quad d_{13}(n)=\left(v_{n, 1}-v_{n-1,3}\right) \\
& r_{02}(n)=\frac{d_{02}(n)}{\Delta_{1}(n)}, \quad r_{13}(n)=\frac{d_{13}(n)}{\Delta_{0}(n+1)} .
\end{aligned}
$$

The ratios of odd parity $r_{01}, r_{10}$ are not considered here. They show a periodic modulation caused by the phase shift induced by the interface between a radiative and convective region, the period being determined by the acoustic radius of the boundary, but the amplitude is sensitive to the ratio of the large separation to the period of the modulation hence changing the envelope structure changes the amplitude. We defer to later later work an analysis of the diagnostic power of such odd parity ratios.

\section{Results}

We constructed a set of models of main sequence stars with masses $0.8,1.0,1.2,1.5,2,10 M_{\odot}$, with initial composition $X=0.7, Z=0.02$, evolved to a central hydrogen abundance of $X_{\mathrm{c}}=0.4$. The equation of state was OPAL EOS2001 (Rogers \& Nayfonov 2001) and the opacities a smooth blend of OPAL GN93 (Iglesias \& Rogers 1996) and Alexander \& Ferguson (1994) tables. Only the major branches of the CN cycle and pp chain were included in the nuclear reaction network. For each model the layers where $r>0.9 R_{0}$ were replaced by a layer with constant polytropic index $m$ with $m=1.5$, $2,2.5,3,3.5,4,4.5,5$, subject to continuity in $r, M_{r}, P, \rho$ at $r=0.9 R_{0}$, and to the boundary condition that $P / \rho$ at the surface had the same value as in the original model. The value of the adiabatic exponent $\Gamma_{1}$ in the polytropic envelope was taken as given by the value $\Gamma_{1}(P / \rho)$ in the reference model. We calculated the oscillation frequencies $v_{n, \ell}$ separations $\Delta_{0}, \Delta_{1}, d_{02}, d_{13}$ and the corresponding ratios $r_{02}(n), r_{13}(n)$ for all the models.

Figure 1 gives the results for the $0.8 M_{\odot}$ models. In the top panel the uppermost set of larger points gives the values of the large separations for the model with polytropic index $m=1.5$ and the lower set of larger points the values for the model with $m=5.0$. The values for models with $m$ between these limits, indicated by the smaller points, decrease essentially monotonically as $m$ increases. The set of points corresponding to the original model are almost identical with those for $m=1.5$ at low frequencies but decrease with increasing frequency becoming almost identical to the values for the model with $m=2$. 

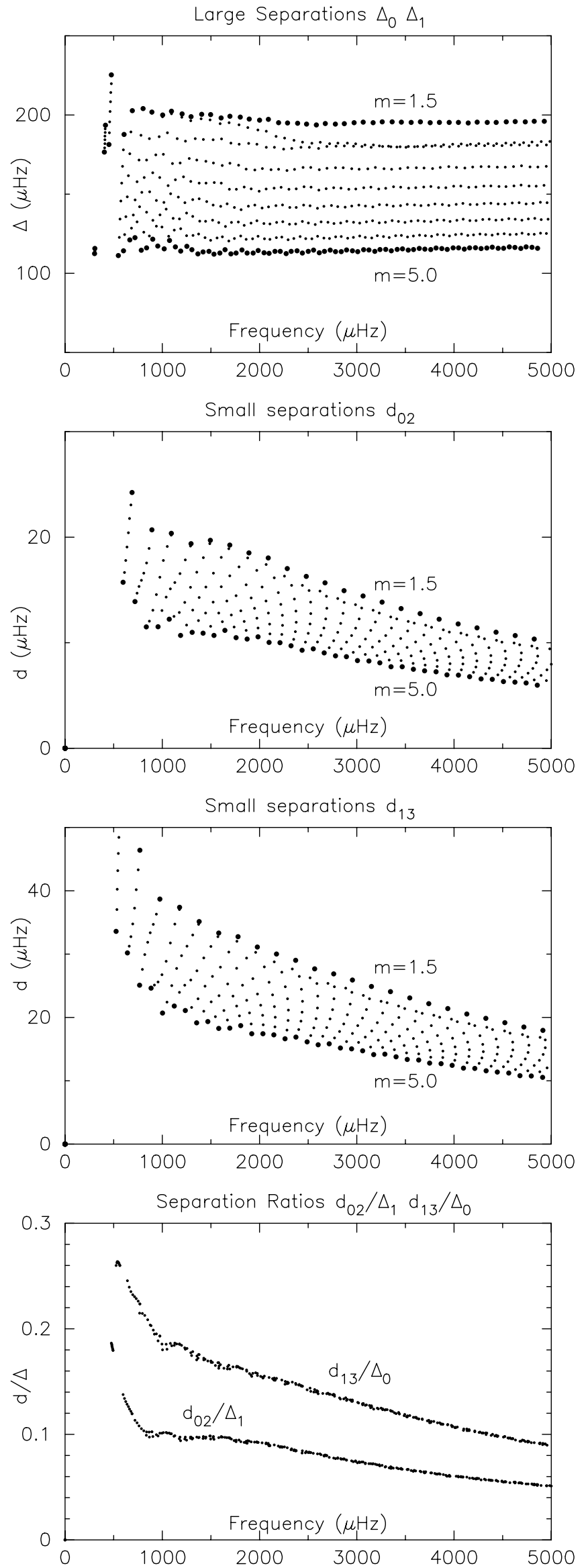

Fig. 1. $0.8 M_{\odot}$ models with polytropic envelopes $r>0.9 R_{0}$.
In the second panel we show the values of the small separation $d_{02}$ for each of the models, again the uppermost set of larger dots correspond to the model with $m=1.5$ and the lower set to the model with $m=5.0$, the values at a given frequency decreasing with increasing $m$. The same applies to the third panel which shows the small separation $d_{13}$. In the bottom panel we show the ratios $r_{02}$ and $r_{13}$ for all the models, these ratios are almost independent of the value of $m$, demonstrating the these ratios are almost independent of the structure of the surface layers of the models and are essentially determined by the interior structure alone. Figure 2 gives similar detailed results for the $10 M_{\odot}$ models, and Fig. 3 the results for the separation ratios for the set of models with $M=1.0,1.2,1.5,2 M_{\odot}$. For all masses the ratios $r_{02}$ and $r_{13}$ are approximately independent of the value of $m$ and hence determined only by the interior structure.

A more dramatic illustration of the independence of the ratios $r_{02}, r_{13}$ to the structure of the outer layers is given by taking the stellar model and simply removing the outer layers altogether, and then determining the ratios for these truncated models. The results of such calculations are shown in Figs. 4 and 5 for models with masses of $0.8,10 M_{\odot}$, which were cut at fractional radii $r_{\mathrm{c}} / R=0.85,0.90,0.95$. The upper panel gives the large separations for the different values of $r_{\mathrm{c}} / R$; the same labelling applies to the values of the small separations in the next 2 panels; the bottom panels show the resulting ratios. Figure 6 shows the ratios for the set of models with masses $1.0,1.2,1.5,2.0 M_{\odot}$. The ratios $r_{02}, r_{13}$ again collapse on to a single function of frequency, the main contribution to the scatter around these curves coming from the values for the largest truncation depth $(0.85)$. We again conclude that these ratios $r_{02}, r_{13}$ are essentially determined by the interior structure alone.

\section{Theory}

The explanation of these results given in $R \& V$ (2003) is in terms of the behaviour of the internal and surface phase shifts. The internal phase shifts $\delta_{\ell}(\omega, t)$ are defined by

$\frac{\omega \psi}{\mathrm{d} \psi / \mathrm{d} t}=\tan \left[\omega t-\ell \pi / 2+\delta_{\ell}(\omega, t)\right]$

where

$\psi=\frac{p^{\prime} r}{(\rho c)^{1 / 2}}, \quad t=\int_{0}^{r} \frac{\mathrm{d} r}{c}$

and $p^{\prime}$ is the Eulerian pressure perturbation. As shown numerically in R\&V (2003), and theoretically in R\&V (1994), the phase shifts $\delta_{\ell}(\omega, t)$ are determined primarily by the structure of the inner regions of the star and are nearly independent of acoustic radius $t$ in the outer regions. 

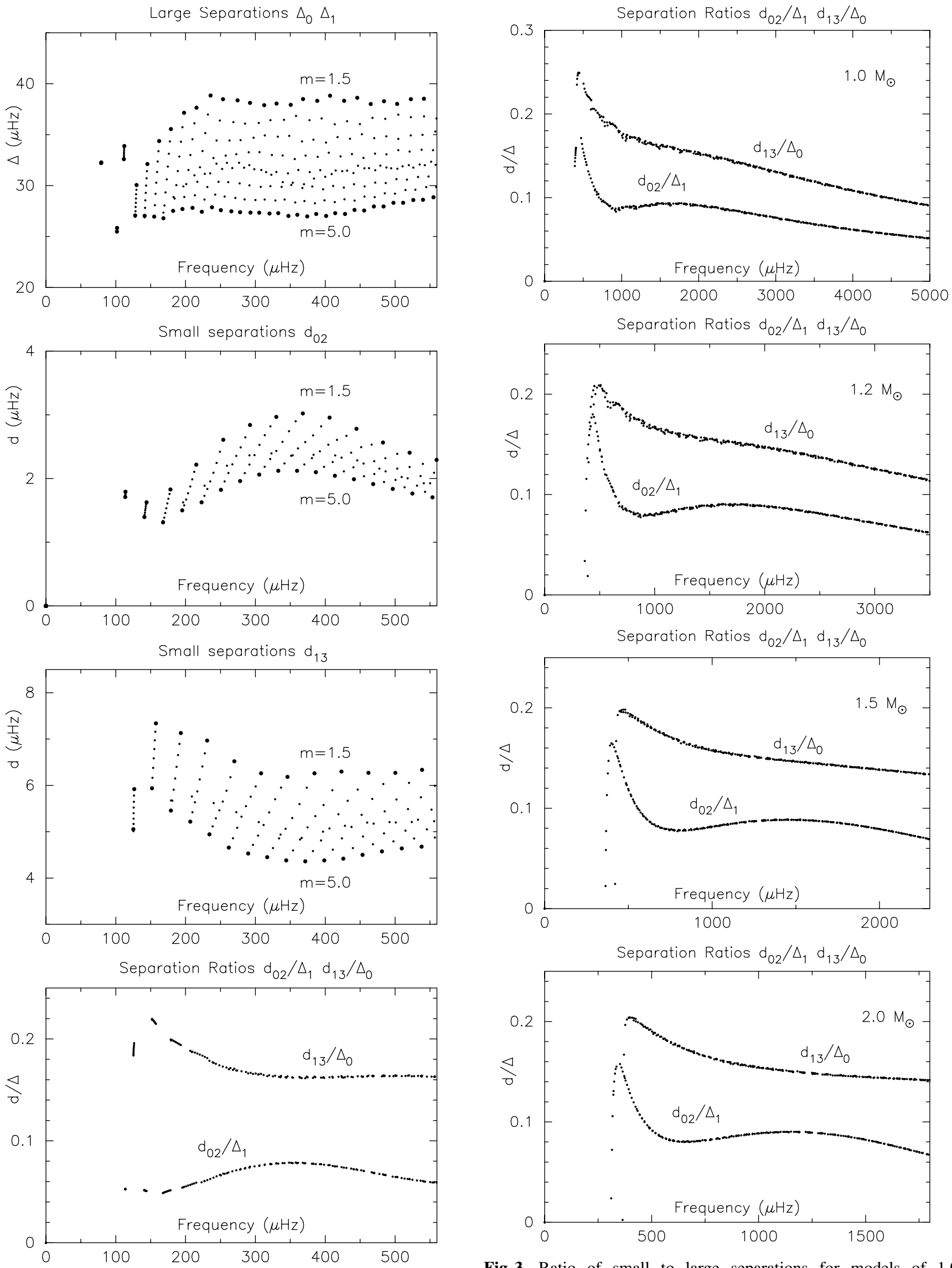

Fig. 2. $10 M_{\odot}$ models with polytropic envelopes $r>0.9 R_{0}$.

Fig. 3. Ratio of small to large separations for models of 1.0, 1.2, 1.5, $2.0 M_{\odot}$ with polytropic envelopes $r>0.9 R_{0}$. 

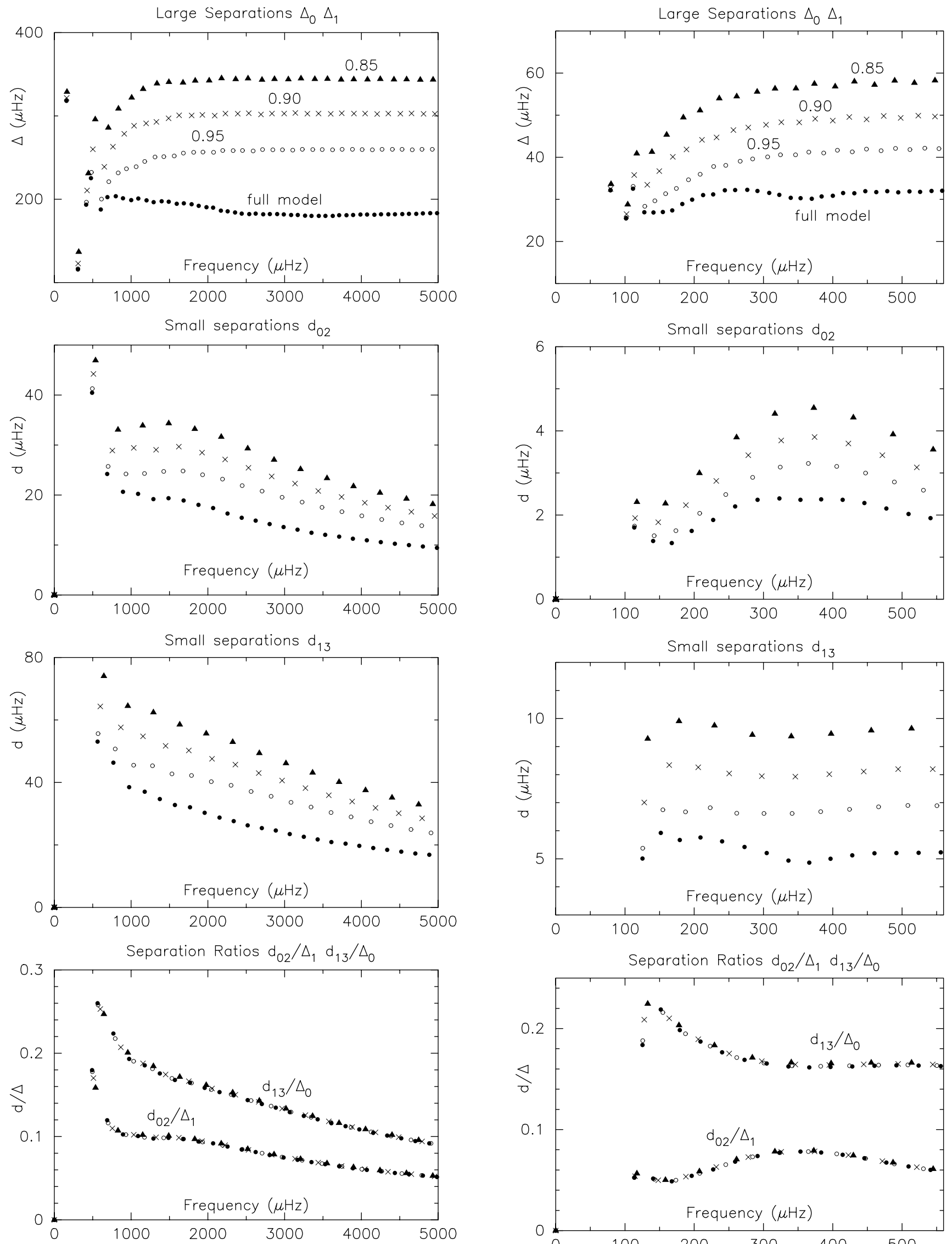

Fig. 4. $0.8 M_{\odot}$ models cut at $r=0.85,0.9,0.95 R_{0}$.

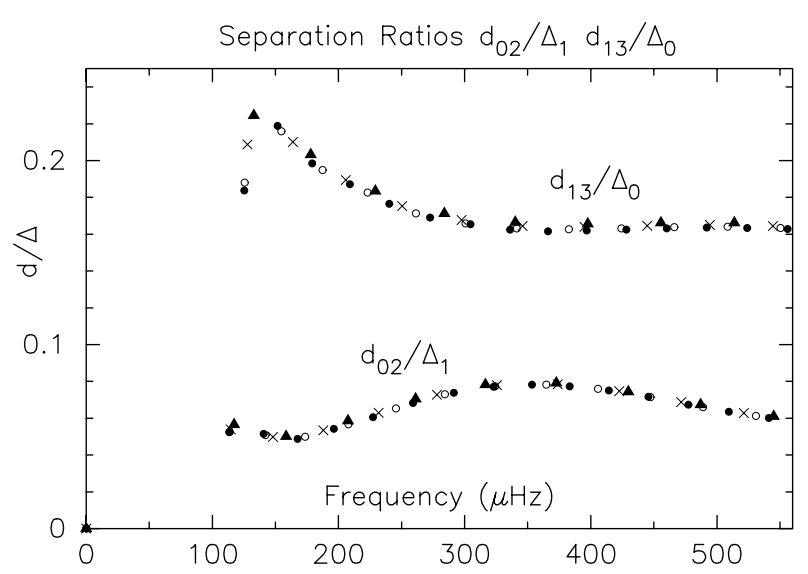

Fig. 5. $10 M_{\odot}$ models cut at $r=0.85,0.9,0.95 R_{0}$. 


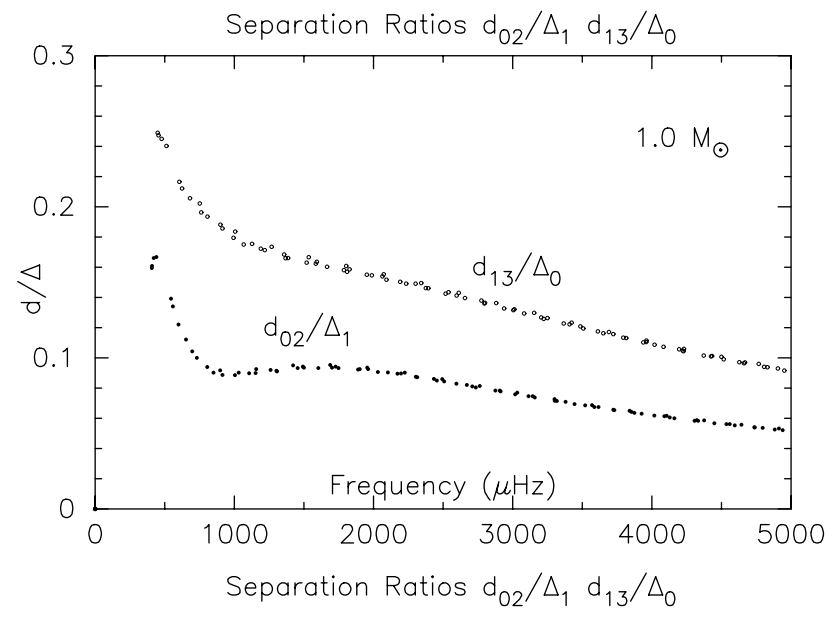

In the outer layers the surface phase shift $\alpha_{\ell}(\omega, t)$ is defined by

$\frac{\omega \psi}{\mathrm{d} \psi / \mathrm{d} t}=-\tan \left[\omega(T-t)-\alpha_{\ell}(\omega, t)\right]$

where, below the surface layers, $\alpha$ is almost independent of degree $\ell$ and of acoustic depth $T-t$, where $T$ is the acoustic radius of the star. Matching the phases in the outer layers gives the Eigenfrequency equation (Roxburgh \& Vorontsov 2000)

$\omega_{n, \ell} T-\ell \pi / 2+\delta_{\ell}\left(\omega_{n, \ell}\right)=n \pi+\alpha\left(\omega_{n, \ell}\right)$.

Using this result to determine the separations gives

$r_{02}=\frac{d_{02}}{\Delta_{1}} \approx \frac{1}{\pi}\left(\delta_{2}-\delta_{0}\right)$
$r_{13}=\frac{d_{13}}{\Delta_{0}} \approx \frac{1}{\pi}\left(\delta_{3}-\delta_{1}\right)$

so these ratios are determined primarily by the internal phase shifts alone.

This analysis is based on the approximation that the surface phase shifts $\alpha_{\ell}\left(\omega_{n, \ell}\right)$ are independent of $\ell$. At large truncation depths this approximation breaks down producing the slight departure of the ratios $r_{02}, r_{13}$ from their values in the reference models as seen in Figs. 4-6.

\section{Discussion}

The goals of extant and upcoming space missions in asteroseismology, MOST (Mathews 1998); COROT (Baglin et al. 1998) and Eddington (Favata et al. 2000), are to achieve accuracies on the measured frequencies of $0.1-0.3 \mu \mathrm{Hz}$. This gives an accuracy in determining the ratios $r_{02}, r_{13}$ which ranges from $10^{-3}$ for small masses up to $10^{-2}$ for the large masses. These ratios are therefore capable of yielding valuable diagnostic information about the internal structure of stars.

Acknowledgements. This work was supported by the UK PPARC under grant PPA/G/S/2003/00137.

\section{References}

Alexander, D. R., \& Ferguson, J. W. 1994, ApJ, 437, 891

Baglin, A., \& the COROT Team 1998, in New Eyes to see inside the Sun and Stars, IAU Symp., 185, 301

Favata, F., Roxburgh, I. W., \& Christensen-Dalsgaard, J., ed. 2000, Eddington, ESA Assessment Study Report, ESA-SCI(2000), 8

Iglesias, C. A., \& Rogers, F. J. 1996, ApJ, 464, 943

Mathews, J. M. 1998, in Structure and Dynamics of the Interior of the Sun and Sun-like stars, SOHO6/GONG98 Workshop, ESA SP-418, 395

Rogers, F. J., \& Nayfonov, A. 2002, ApJ, 576, 1064

Roxburgh, I. W. 2004, in Stellar Structure and Habitable Planet Finding, ed. F. Favata, S. Aigrain, \& A. Wilson, ESA SP-538, 23

Roxburgh, I. W., \& Vorontsov, S. V. 1994, MNRAS, 267, 297

Roxburgh, I. W., \& Vorontsov, S. V. 2000, MNRAS, 317, 141

Roxburgh, I. W., \& Vorontsov, S. V. 2003, A\&A, 411, 215

Fig. 6. Ratio of small to large separations for models of 1.0, 1.2, 1.5, $2.0 M_{\odot}$ cut at $r=0.85,0.9,0.95 R_{0}$. 